\title{
Bounds on the Action Degree of Groups
}

\author{
${ }^{1}$ S. Alrehaili and ${ }^{2} \mathrm{C}$. Beddani ${ }^{*}$ \\ ${ }^{1,2}$ Department of Mathematics, College of Science, Taibah University, \\ Madinah, Saudi Arabia \\ ${ }^{1}$ Department of Mathematical Sciences, Universiti Teknologi Malaysia \\ 81310 UTM Johor Bahru, Malaysia \\ *Corresponding author: chbeddani@gmail.com
}

Article history

Received: 4 October 2018

Received in revised form: 6 April 2019

Accepted: 7 May 2019

Published online: 1 August 2019

\begin{abstract}
The commutativity degree is an invariant used to measure the probability that two arbitrarily chosen group elements commute. This concept was generalized by many authors in different ways. One of these generalizations involves the action degree of finite groups, which is the probability that a random group element fixes a random element from a nonempty set. In this paper, we present some properties and important inequalities about the action degree and give a relationship between the action degree of a finite group and that of the kernel subgroup of this action. In addition, we show that the action degree play an important role to determine some information about the cardinality of the set of all fixed points. On the other hand, we introduce in this paper a new definition for the action degree of finitely generated groups. This definition generalizes another recent definition of the commutativity degree of finitely generated groups. Furthermore, some boundaries with respect to this degree are presented.
\end{abstract}

Keywords Group theory; action degree; commutativity degree of groups; finite groups; infinite groups.

\section{Mathematics Subject Classification 20P05, 58E40.}

\section{Introduction}

There has been a growing interest in recent years on the use of the probability in group theory. One of the most important aspects which have been studied extensively is the probability that a selected random pair of elements $(x, y)$ in a group $G$ commute, denoted as $c d(G)$. In fact, the determination of the commutativity degree of a group yields a wealth of information about the group's structure and properties. This concept was introduced in 1968 by Erdos and Turan [1], where they worked on symmetric groups.

Gustafson [2] showed that this probability can be computed using conjugacy classes and MacHale [3] computed it using 0,1-Table. Numerous researches have been done on the commutativity degree and many results have been achieved. In 1975, Sherman [4] defined the 
probability that a random element from a group $G$ fixes a random element from a non-empty set $S$. We refer to this probability as the action degree of groups, $a d_{S}(G)$. Sherman [4] studied the probability that an automorphism fixes a group element, which gives commutativity degree in a special case. He considered only the case for which $G$ is a finite abelian group and $A$ is its group of automorphisms.

Omer et al. [5] generalized the commutativity degree by introducing the probability of an element $g$ in $G$ to fix a set $X$ and $G$ acts on the set of all subset of commuting elements in $G$ of size 2. Moreover, the probability is used on different group structures namely dihedral group, quaternion group and alternating group. The probability was found for some symmetric groups in [6] in the case that a group acts on a set regularly and by conjugation. In 2015, El-sanfaz et al. [7] found the probability that a group element fixes a set under regular action for metacyclic 2-groups of positive type of nilpotency class of at least three.

In the first part of this paper, the general definition of action degree (the probability that a random element from a group $G$ fixes a random element from a non-empty set $S$ ) is considered where some inequalities and bounds on the action degree of finite groups are determined.

In 2017, Antolin et al. [8] defined the commutativity degree of finitely generated groups and proved that there is a generalization of Gustafson's result on the bound of commutativity degree of finite groups. Inspired by their work, we generalize the definition of the action degree of finite groups and define the action degree of finitely generated groups. Furthermore, some bounds and limiting conditions on this degree are obtained.

\section{Some Basic Concepts}

The aim of this section is to briefly recall some definitions and preparatory results that are needed throughout this article.

In the following we recall the formal definitions of the commutativity degree and some of its generalizations which are related to this research and we begin with the concept of commutativity degree.

\section{Definition $1[2]$}

Let $G$ be a group, the commutativity degree is the probability that two random elements $(x, y)$ in $G$ commute, defined as:

$$
c d(G)=\frac{|\{(x, y) \in G \times G \mid x y=y x\}|}{|G|^{2}} .
$$

The action degree of finite groups is defined as follows:

\section{Definition $2[4]$}

The probability that a random element from a group $G$ fixes a random element from the non-empty set $S$ is defined as:

$$
a d_{S}(G)=\frac{|\{(x, s) \in G \times S \mid x s=s\}|}{|S| \cdot|G|} .
$$

\section{Remark:}

Let $G$ be a finite group acts on a finite set $S$. 
1. The probability $a d_{S}(G)=1$ if and only if $g s=s$ for all $g \in G$ and $s \in S$.

2. If $G$ acts on itself by conjugation, $g s=g s g^{-1}$, then in this case, $a d_{S}(G)=c d(G)$, the commutativity degree of $G$.

It is useful to recall some basic concepts regarding the action of groups. Given a finite group $G$ acting on a finite set $S$, the stabilizer of an element $s \in S$ is the subgroup $G_{s}$ of $G$ defined by $G_{s}=\{g \in G \mid g s=s\}$. By definition of the stabilizer, it is clear that the action degree can be defined as:

$$
\operatorname{ad}_{S}(G)=\frac{\sum_{s \in S}\left|G_{s}(G)\right|}{|S| \cdot|G|}
$$

The orbit of an element $s$ in $S$ is the subset $o(s)$ of $G$ defined as $o(s)=\{g s \mid g \in G\}$. The relation $\sim$ on $S$ defined by $s \sim t$ if $s=g t$ for some $g \in G$ is an equivalent relation. Furthermore, the equivalent class of any $s \in S$ is the orbit $o(s)$. The number of distinct equivalent classes of this relation is denoted by $k_{s}(G)$.

The following is a result by Sherman [4] that can simplify the computation of the action degree. It is also needed to prove Corollary 1.

\section{Proposition 1 [4]}

Let $G$ be a finite group acts on a finite set $S$. Then $a_{s}(G)=\frac{k_{s}(G)}{|S|}$, where $k_{s}(G)$ denotes the number of distinct equivalent classes.

The focus in Sherman's research [4] was on the probability that an automorphism fixes a group element. He considered $P_{A}(G)$, where $G$ is a finite abelian group and $A$ is its group of automorphisms where he obtained some bounds and limiting conditions on this probability. In this paper, we provide some bounds and relations between the action degree of groups, the set of fixed points of the action and the kernel of action. Meanwhile, the class equation of group action is used to prove some of these relations. We recall these concepts in the following.

If $G$ is a finite group that acts on a finite set $S$, the set

$$
S_{0}(G)=\{s \in S \quad \mid g s=s \text { for all } g \in G\}
$$

is called the fixed point set of the action. It may also be viewed as the union of all single element orbits. Suppose a group $G$ is acting on a finite set $S$. If $\left\{o\left(s_{1}\right), o\left(s_{2}\right), \ldots, o\left(s_{k}\right)\right\}$ is the set of distinct orbits of $G$ on $S$. Then

$$
|S|=\sum_{i=1}^{k}\left|o\left(s_{i}\right)\right| .
$$

If $I=\{1 \leq i \leq n$ such that $|o(s)|>1\}$, then

$$
|S|=\left|S_{0}(G)\right|+\sum_{i=1}^{k}\left|o\left(s_{i}\right)\right| .
$$

Let $G$ be a finite group that acts on a finite set $S$, the set

$$
Z_{s}(G)=\{g \in G \mid g s=s \quad \forall s \in S\}
$$


is known as the kernel of action and it is a normal subgroup of $G$.

One main objective of this research is to determine some bounds on the probability that a random element from a group $G$ fixes a random element from the non-empty set $S$ that was presented by Sherman [4]. As mentioned earlier, we refer to this probability by the action degree of groups.

The other main objective of this article is to extend the work on action degree of finite groups to the finitely generated groups. Thus, the following part presents some basic concepts and earlier studies on the degree of commutativity of finitely generated groups.

Let $G$ be a group and $S$ a generating set for $G$. The set of inverses of elements of $S$ is denoted by $S^{-1}$. The length function $l_{S}: G \rightarrow N$ gives the smallest integer $n$ such that there exist $s_{1}, s_{2}, \ldots, s_{n} \in S \cup S^{-1}$ with $g=s_{1}, s_{2}, \ldots, s_{n}$. It is then often written $|g|=n$. The notion of the growth of a finitely generated group was introduced by Schwarz [9] and independently by Milnor et al. [10]. The definition is given as follows: Let $G$ be a finitely generated group and $S$ a finite set of generators for $G$. The growth function of the group $G$ with respect to $S$, denoted as $B_{S}(n)$ is the set of elements in $G$ that can be written as a word on $S$ having length at most $n$. If $S$ and $T$ are two finite generating sets for $G$, then the growth functions with respect to $S$ and $T$ are equivalent. So for each group $G$ the growth type, defined to be the equivalence class of one of its growth functions is distinct and independent of any generating set.

Some bounds on the action degree of finitely generated groups in the current research are only applied to groups of subexponential growth. Accordingly, this concept needs to be clarified. By Grigorchuk [11], the $\operatorname{limit}_{n \rightarrow \infty}\left|B_{S}(n)\right|^{\frac{1}{n}}$ always exists and it is called the exponential growth rate of $G$ with respect to $S$. Moreover, this limit depends on the set of generators $S$ but the fact of being 1 or larger than 1 does not depend on $S$. Therefore, it is an invariant of the group $G$. A group is said to have exponential growth when its exponential growth rate is larger than one, and subexponential growth when the exponential growth rate is one.

In 2017, Antolin et al. [8] defined the commutativity degree of finitely generated groups as follows:

Definition 3 [8] Let $G$ be a finitely generated group and $X$ a finite generating set. The degree of commutativity of $G$ with respect to $X$, denoted $c d_{X}(G)$, is defined as:

$$
c d_{X}(G)=\lim _{n \rightarrow \infty} \sup \frac{\left|\left\{(g, h) \in\left(B_{X}(n)\right)^{2} \mid g h=h g\right\}\right|}{\left|B_{X}(n)\right|^{2}}
$$

where $B_{X}(n)=\left\{g \in G: l_{X}(g) \leq n\right\}$ for every generating set $X$ of $G$.

The next lemma is needed to prove some bounds on the action degree of finitely generated groups.

Lemma 1 [12] Let $G$ be a finitely generated group with subexponential growth, and let $X$ be a finite generating set for $G$. For every finite index subgroup $H$ of $G$ and every $g \in G$, we have:

$$
\lim _{n \rightarrow \infty} \frac{\left|g H \cap B_{X}(n)\right|}{\left|B_{X}(n)\right|}=\frac{1}{[G: H]} .
$$

The present paper introduces a similar definition of the action degree of finitely generated groups. Further, some useful bounds and inequalities regarding this probability are provided. 


\section{Results and Discussion}

The main results of this paper are presented in this section where some inequalities and limiting conditions on the action degree of finite groups are provided. Moreover, the definition and some properties of the action degree of finitely generated groups are presented.

\subsection{Action Degree of Finite Groups}

Given a finite group $G$ acting on a finite set $S$, we define a set $A_{S}(G)$ that consists of all ordered pairs $(x, s)$ in $G \times S$ where the element $x \in G$ fixes $s \in S$,precisely, $A_{S}(G)=$ $\{(x, s) \in G \times S \mid x s=s\}$.

Therefore

$$
\operatorname{ad}_{S}(G)=\frac{A_{S}(G)}{|S| \cdot|G|}
$$

Throughout this paper, we use this formula or the formula of the stabilizers of elements to prove our results.

Lemma 2 Let $G$ be a finite group that acts on a finite set $S$. Then

$$
\operatorname{ad}_{S}(G) \geq \frac{1}{|G|} \text {. }
$$

Proof Since $1 s=s$ for all $s \in S$, then

$$
\{1\} \times S \subseteq A_{S}(G) .
$$

Therefore

$$
|S| \leq\left|A_{S}(G)\right|
$$

Dividing by $|S| \cdot|G|$, we get the result.

Proposition 2 Let $G$ be a finite group that acts on a finite set $S$ and $H$ a subgroup of $G$. Then:

$$
a d_{S}(H) \leq[G: H] \quad a d_{S}(G) .
$$

Proof For all $s \in S$, we have

$$
G_{s}(H) \subseteq G_{s}(G)
$$

Then,

$$
\sum_{s \in S}\left|G_{s}(H)\right| \leq \sum_{s \in S}\left|G_{s}(G)\right|
$$

From this inequality and since $|H|=|G|[G: H]^{-1}$, we get

$$
\frac{1}{|H|} \sum_{s \in S}\left|G_{s}(H)\right| \leq \frac{[G: H]}{|G|} \sum_{s \in S}\left|G_{s}(G)\right| .
$$

That means

$$
a d_{S}(H) \leq[G: H] \quad a d_{S}(G)
$$

Applying Proposition 1, we obtain the following corollary: 
Corollary 1 Let $G$ be a finite group that acts on a finite set $S$ and $H$ a subgroup of $G$. Then

$$
k_{s}(H) \leq[G: H] \quad k_{s}(G) .
$$

This corollary is a generalization of a result proved by Ernest [13] in the case when $k(G)$ is the number of conjugacy classes of $G$.

Proposition 3 Let $H$ and $K$ be two finite groups acting respectively on finite sets $S_{1}$ and $S_{2}$ such that $S_{1} \cap S_{2}=\emptyset$. Then

$$
a d_{S_{1} \times S_{2}}(H \times K)=a d_{S_{1}}(H) \cdot a d_{S_{2}}(K) .
$$

Proof First recall that the group $H \times K$ acts naturally on $S_{1} \times S_{2}$ via:

$$
(h, k)\left(s_{1}, s_{2}\right)=\left(h s_{1}, k s_{2}\right) .
$$

We know that

$$
|H \times K|=|H| \cdot|K|
$$

and

$$
\left|\left(S_{1} \times S_{2}\right)\right|=\left|S_{1}\right| \cdot\left|S_{2}\right| .
$$

To prove

$$
a d_{S_{1} \times S_{2}}(H \times K)=a d_{S_{1}}(H) \cdot a d_{S_{2}}(K),
$$

we need to show that:

$$
\left|A_{S_{1} \times S_{2}}(H \times K)\right|=\left|A_{S_{1}}(H)\right| \cdot\left|A_{S_{2}}(K)\right| .
$$

Consider the following function

$$
\psi: A_{S_{1} \times S_{2}}(H \times K) \rightarrow A_{S_{1}}(H) \times A_{S_{2}}(K)
$$

defined by:

$$
\psi\left((h, k),\left(s_{1}, s_{2}\right)\right)=\left(\left(h, s_{1}\right),\left(k, s_{2}\right)\right) .
$$

Remark that this function is well-defined and it is a bijection. Then

$$
\begin{aligned}
\left|A_{S_{1} \times S_{2}}(H \times K)\right|= & \left|A_{S_{1}}(H) \times A_{S_{2}}(K)\right| \\
& =\left|A_{S_{1}}(H)\right| \cdot\left|A_{S_{2}}(K)\right| .
\end{aligned}
$$

So,

$$
\begin{aligned}
a d_{S_{1} \times S_{2}}(H \times K) & =\frac{\left|A_{S_{1} \times S_{2}}(H \times K)\right|}{\left|S_{1} \times S_{2}\right| \cdot|H \times K|} \\
& =\frac{\left|A_{S_{1}}(H)\right| \cdot\left|A_{S_{2}}(K)\right|}{\left|S_{1}\right| \cdot\left|S_{2}\right| \cdot|H| \cdot|K|} \\
& =\left(\frac{\left|A_{S_{1}}(H)\right|}{\left|S_{1}\right| \cdot|H|}\right)\left(\frac{\left|A_{S_{2}}(K)\right|}{\left|S_{2}\right| \cdot|K|}\right) \\
& =a d_{S_{1}}(H) \cdot a d_{S_{2}}(K) .
\end{aligned}
$$


Before proceeding to the next theorem, recall that the kernel of action

$$
Z_{s}(G)=\{g \in G \mid g s=s \quad \forall s \in S\},
$$

is a normal subgroup of $G$. In addition, $G / Z_{s}(G)$ acts on the set $S$ by $(g H) s=g s$ and this action is well defined.

Before proceeding to the next theorem, recall that the kernel of action

$$
Z_{s}(G)=\{g \in G \mid g s=s \quad \forall s \in S\},
$$

is a normal subgroup of $G$. In addition, $G / Z_{s}(G)$ acts on the set $S$ by $(g H) s=g s$ and this action is well defined.

Theorem 1 Let $G$ be a finite group that acts on a finite set $S$ and $H=Z_{s}(G)$. Then ad $d_{S}(G)=$ $a d_{S}(G / H)$.

Proof Since $g s=s$ if and only if $g H s=s$, we have:

$$
\left|A_{S}(G)\right|=|H| \cdot\left|A_{S}(G / H)\right| .
$$

Then

$$
\begin{aligned}
a d_{S}(G / H) & =\frac{\left|A_{S}(G / H)\right|}{|G / H| \cdot|S|} \\
& =\frac{\left|A_{S}(G)\right|}{|H| \cdot|G / H| \cdot|S|} \\
& =\frac{\left|A_{S}(G)\right|}{|G| \cdot|S|} \\
& =a d_{S}(G) .
\end{aligned}
$$

Proposition 4 Let $G$ be a finite group that acts on a finite set $S$. Then

$$
a d_{S}(G) \geq \frac{\left|Z_{s}(G)\right|}{|G|} .
$$

Proof Recall that

$$
A_{S}(G)=\{(x, s) \in G \times S \mid x s=s\} .
$$

Clearly, for all $g \in Z_{s}(G)$, we have $\{g\} \times S \subseteq A_{S}(G)$ which shows that:

$$
Z_{s}(G) \times S \subseteq A_{S}(G) \text {. }
$$

Therefore

$$
\begin{aligned}
a d_{S}(G) & \geq \frac{\left|Z_{s}(G) \times S\right|}{|S| \cdot|G|} \\
& =\frac{\left|Z_{s}(G)\right| \cdot|S|}{|S| \cdot|G|} \\
& =\frac{\left|Z_{s}(G)\right|}{|G|} .
\end{aligned}
$$


Proposition 5 Let $G$ be a finite group that acts on a finite set $S$. Then

$$
a d_{S}(G) \leq \frac{1}{2}+\frac{\left|S_{0}(G)\right|}{2|S|} .
$$

Proof Consider the class equation:

$$
|S|=\left|S_{0}(G)\right|+\sum_{i=\left|S_{0}(G)\right|+1}^{k_{s}(G)}\left|o\left(s_{i}\right)\right| .
$$

Then

$$
|S| \geq\left|S_{0}(G)\right|+2\left(k_{s}(G)-\left|S_{0}(G)\right|\right) .
$$

Therefore

$$
|S| \geq 2 k_{s}(G)-\left|S_{0}(G)\right|
$$

Solving for $k_{s}(G)$ yields

$$
k_{s}(G) \leq \frac{|S|+\left|S_{0}(G)\right|}{2} .
$$

Therefore

$$
a d_{S}(G) \leq \frac{1}{2}+\frac{\left|S_{0}(G)\right|}{2|S|} .
$$

Proposition 6 Let $G$ be a finite group that acts on a finite set $S$. Then

$$
a d_{S}(G) \geq \frac{\left|S_{0}(G)\right|}{|S|} .
$$

Proof Let $t$ be a fixed point of this action. Then $(x, t) \in A_{S}(G)$ for all $x \in G$. Hence

$$
G \times S_{0}(G) \subseteq A_{S}(G) .
$$

From this we can show that:

$$
a d_{S}(G) \geq \frac{\left|S_{0}(G)\right|}{|S|} .
$$

Corollary 2 If $a d_{S}(G)>\frac{1}{2}$, then there is at least one fixed point.

Proof Assume that $a d_{S}(G)>\frac{1}{2}$. By using the inequality (1), we get

$$
\frac{\left|S_{0}(G)\right|}{|S|}>0
$$

This shows that $\left|S_{0}(G)\right|>0$, and hence the set $S_{0}(G)$ is not empty.

Remark that if the action has a fixed point, then $a d_{S}(G)$ is not necessary greater than $\frac{1}{2}$, which means that the opposite of Corollary (2) is not always true. For an example, let $G$ be a group acting on itself by conjugation. Then the action of $G$ on itself in this case is the commutativity degree of $G$ and $s g=g s g^{-1}=s \quad \forall g \in G$. Then $g s=s g \quad \forall g \in G$. Therefore $s \in Z(G)$ is a fixed point of this action while the action degree (commutativity degree in this case) of the group can be less than $\frac{1}{2}$ as in the symmetric group $S_{4}$ for instance. 
Theorem 2 Let $G$ be a finite group that acts on a finite set $S$. Let $a=\frac{\left|Z_{s}(G)\right|}{|G|}$ and $b=$ $\frac{\left|S_{0}(G)\right|}{|S|}$. Then

$$
a d_{S}(G) \geq a+b-a b .
$$

Proof We know that:

$$
\left(Z_{s}(G) \times S\right) \cup\left(G \times S_{0}(G)\right) \subseteq A_{S}(G) .
$$

So

$$
\left|A_{S}(G)\right| \geq\left|Z_{s}(G) \times S\right|+\left|G \times S_{0}(G)\right|-\left|Z_{s}(G) \times S_{0}(G)\right| .
$$

Therefore

$$
\left|A_{S}(G)\right| \geq\left|Z_{s}(G)\right| \cdot|S|+|G| \cdot\left|S_{0}(G)\right|-\left|Z_{s}(G)\right| \cdot\left|S_{0}(G)\right|
$$

Finally

$$
a d_{S}(G) \geq \frac{\left|Z_{s}(G)\right|}{|G|}+\frac{\left|S_{0}(G)\right|}{|S|}-\left(\frac{\left|Z_{s}(G)\right|}{|G|}\right)\left(\frac{\left|S_{0}(G)\right|}{|S|}\right) .
$$

In the following, the definition of the action degree of finitely generated groups is presented along with some bounds and inequalities.

\subsection{Action Degree of Finitely Generated Groups}

So far, this paper has focused on finite groups. The following part moves on to finitely generated groups. We begin with the definition of the action degree of finitely generated groups.

Definition 4 Let $G$ be a finitely generated group and $X$ a finite generating set of $G$. Assume that $G$ acts on a finite set $S$. We define the action degree of $G$ with respect to $X$, denoted as $a d_{S}(G, X)$ as:

$$
a d_{S}(G, X)=\lim _{n \rightarrow \infty} \sup \frac{\left|\left\{(g, s) \in B_{X}(n) \times S \mid g s=s\right\}\right|}{\left|B_{X}(n)\right| \cdot|S|}
$$

where $B_{X}(n)=\left\{g \in G: l_{X}(g) \leq n\right\}$ for every generating set $X$ of $G$.

If $G$ acts on itself by conjugation $g s=g s g^{-1}$, then:

$$
c d_{X}(G)=\lim _{n \rightarrow \infty} \sup \frac{\left|\left\{(g, h) \in\left(B_{X}(n)\right)^{2} \mid g h=h g\right\}\right|}{\left|B_{X}(n)\right|^{2}}
$$

and this is the degree of commutativity of infinite groups presented by Antolin et al. in [8].

Proposition 7 If $G$ is a finite group, then $a_{S}(G, X)=a d_{S}(G)$ for every generating set of $G$. 
Proof Assume that $G$ is finite, then there exists $n_{0}$ such that for all $n \geq n_{0}, B_{X}(n)=G$. So

$$
\frac{\left|\left\{(g, s) \in B_{X}(n) \times S \mid g s=s\right\}\right|}{\left|B_{X}(n)\right| \cdot|S|}=\frac{|\{(g, s) \in G \times S \mid g s=s\}|}{|G| \cdot|S|},
$$

for all $n \geq n_{0}$. Passing to limit when $n \rightarrow \infty$, we get $a d_{S}(G, X)=a d_{S}(G)$.

Note that if $X$ and $Y$ are two generating sets of $G$, then $a d_{S}(G, X)=a d_{S}(G, Y)$. This follows directly from the fact that the growth functions with respect to $X$ and $Y$ are equivalent. Now, let us determine some inequalities on the action degree of finitely generated groups.

Theorem 3 Let $S_{0}(G)=\{s \in S \mid g s=s$ for all $g \in G\}$. Then

$$
\operatorname{ad}_{S}(G, X) \geq \frac{\left|S_{0}\right|}{|S|} \text {. }
$$

Proof Consider the set

$$
A_{S}(G, X)_{n}=\left\{(g, s) \in B_{X}(n) \times S \mid g s=s\right\} .
$$

Clearly

$$
B_{X}(n) \times S_{0}(G) \subseteq A_{S}(G, X)_{n} .
$$

So

$$
\left|A_{S}(G, X)_{n}\right| \geq B_{X}(n) \times S_{0}(G)=\left|B_{X}(n)\right| \cdot\left|S_{0}(G)\right| .
$$

Therefore, for all $n \geq 1$.

$$
\frac{\left|A_{S}(G, X)_{n}\right|}{|S| \cdot\left|B_{X}(n)\right|} \geq \frac{\left|B_{X}(n)\right| \cdot\left|S_{0}(G)\right|}{|S| \cdot\left|B_{X}(n)\right|}=\frac{\left|S_{0}(G)\right|}{|S|} .
$$

Passing to limit when $n \rightarrow \infty$, we obtain the result.

Theorem 4 Let $G$ be a finitely generated group that acts on a finite set $S$ and let $X$ be a finite generating set for $G$. Suppose that $G$ has subexponential growth. Then for every finite index normal subgroup $H$ of $G$ contained in $Z_{s}(G)$,

$$
a d_{S}(G, X) \leq a d_{S}(G / H) .
$$

Proof Since $H$ is a finite index normal subgroup of $G$, then

$$
\operatorname{ad}_{S}(G / H)=\frac{\left|A_{S}(G / H)\right|}{|S| \cdot[G: H]} .
$$

Then the set $A_{S}(G / H)$ can be written as:

$$
A_{S}(G / H)=\bigcup_{i=1}^{r}\left\{\left(g_{i} H, s_{i}\right)\right\},
$$

for some $g_{i} \in G$ and $s_{i} \in S$. Consider the set

$$
A_{S}(G, X)_{n}=\left\{(g, s) \in B_{X}(n) \times S \mid g s=s\right\} .
$$

Remark that for every element $(g, s) \in A_{S}(G, X)_{n}$, we have:

$$
(g, s) \in\left(g H \cap B_{X}(n)\right) \times\{s\} .
$$


Since $(g H, s)$ is an element in $A_{S}(G / H)$, we obtain

$(g, s) \in\left(g_{i} H \cap B_{X}(n)\right) \times\left\{s_{i}\right\}$, for some $i=1, \ldots, r$. This shows that

$$
A_{S}(G, X)_{n} \subseteq \bigcup_{i=1}^{r}\left(g_{i} H \cap B_{X}(n)\right) \times\left\{s_{i}\right\} .
$$

Therefore

$$
\left|A_{S}(G, X)_{n}\right| \leq \sum_{i=1}^{r}\left|g_{i} H \cap B_{X}(n)\right| .
$$

Hence

$$
\begin{aligned}
\frac{\left|A_{S}(G, X)_{n}\right|}{|S| \cdot\left|B_{X}(n)\right|} & \leq \frac{\sum_{i=1}^{r}\left|g_{i} H \cap B_{X}(n)\right|}{|S| \cdot\left|B_{X}(n)\right|} \\
& \leq \frac{1}{|S|} \sum_{i=1}^{r} \frac{\left|g_{i} H \cap B_{X}(n)\right|}{\left|B_{X}(n)\right|} .
\end{aligned}
$$

Passing to limit when $n \rightarrow \infty$ we get by using Lemma 1 :

$$
\begin{aligned}
a d_{S}(G, X) & \leq \frac{1}{|S|} \sum_{i=1}^{r} \lim _{n \rightarrow \infty} \frac{\left|g_{i} H \cap B_{X}(n)\right|}{\left|B_{X}(n)\right|} \\
& \leq \frac{1}{|S|} \sum_{i=1}^{r} \frac{1}{[G: H]} \\
& =\frac{\left|A_{S}(G / H)\right|}{|S| \cdot[G: H]} \\
& =a d_{S}(G / H) .
\end{aligned}
$$

\section{Conclusion}

In this paper, the probability that an element of a group fixes a set is further studied for finite groups. Several results concerning the action degree are presented along with some limiting conditions and inequalities. Moreover, the work on action degree of finite groups is extended to finitely generated groups which generalizes previous results on the commutativity degree of finitely generated groups.

\section{References}

[1] Erdős, P. and Turán, P. On some problems of a statistical group-theory. iv. Acta Mathematica Hungarica. 1968. 19(3-4): 413-435.

[2] Gustafson, W. H. What is the probability that two group elements commute? The American Mathematical Monthly. 1973. 80(9): 1031-1034. ISSN 0002-9890.

[3] MacHale, D. How commutative can a non-commutative group be? The Mathematical Gazette. 1974. 58(405): 199-202. ISSN 0025-5572.

[4] Sherman, G. What is the probability an automorphism fixes a group element? The American Mathematical Monthly. 1975. 82(3): 261-264. ISSN 0002-9890. 
[5] Omer, S. M. S., Sarmin, N. H., Erfanian, A. and Moradipour, K. The probability that an element of a group fixes a set and the group act on set by conjugation. International Journal of Applied Mathematics and Statistics. 2013. 32(2): 111-117.

[6] Omer, S. M. S., Sarmin, N. H. and Erfanian, A. The probability that an element of a symmetric group fixes a set and its application in graph theory. World Applied Sciences Journal. 2013. 27(12): 1637-1642. ISSN 1818-4952.

[7] El-Sanfaz, M. A., Sarmin, N. H. and Omer, S. M. S. On the probability that a group element fixes a set and its generalized conjugacy class graph. International Journal of Mathematical Analysis. 2015. 9(4): 161-167.

[8] Antolín, Y., Martino, A. and Ventura, E. Degree of commutativity of infinite groups. Proceedings of the American Mathematical Society. 2017. 145(2): 479-485. ISSN 00029939.

[9] Švarc, A. S. A volume invariant of coverings. In Dokl. Akad. Nauk SSSR (NS). 1955. 105: $32-34$.

[10] Milnor, J. et al. A note on curvature and fundamental group. Journal of Differential Geometry. 1968. 2(1): 1-7.

[11] Grigorchuk, R. and De La Harpe, P. On problems related to growth, entropy, and spectrum in group theory. Journal of Dynamical and Control Systems. 1997. 3(1): 51-89.

[12] Burillo, J. and Ventura, E. Counting primitive elements in free groups. Geometriae Dedicata. 2002. 93(1): 143-162.

[13] Ernest, J. A. Central intertwining numbers for representations of finite groups. Transactions of the American Mathematical Society. 1961. 99(3): 499-508. 